\title{
Growth and characterization of TiN/SiN(001) superlattice films
}

\author{
Hans Söderberg ${ }^{\text {a) }}$ and Magnus Odén \\ Division of Engineering Materials, Luleå University of Technology, SE-971 87 Luleå, Sweden \\ Axel Flink, Jens Birch, Per O.Å. Persson, Manfred Beckers, and Lars Hultman \\ Thin Film Physics Division, Linköping University, SE-581 83 Linköping, Sweden
}

(Received 5 February 2007; accepted 20 August 2007)

\begin{abstract}
We report the layer structure and composition in recently discovered $\mathrm{TiN} / \mathrm{SiN}(001)$ superlattices deposited by dual-reactive magnetron sputtering on $\mathrm{MgO}(001)$ substrates. High-resolution transmission electron microscopy combined with Z-contrast scanning transmission electron microscopy, $\mathrm{x}$-ray reflection, diffraction, and reciprocal-space mapping shows the formation of high-quality superlattices with coherently strained cubic TiN and SiN layers for SiN thickness below 7-10 A. For increasing SiN layer thicknesses, a transformation from epitaxial to amorphous $\operatorname{SiN}_{x}(x \geqslant 1)$ occurs during growth. Elastic recoil detection analysis revealed an increase in nitrogen and argon content in $\mathrm{SiN}_{x}$ layers during the phase transformation. The oxygen, carbon, and hydrogen contents in the multilayers were around the detection limit $(\sim 0.1$ at.\%) with no indication of segregation to the layer interfaces. Nanoindentation experiments confirmed superlattice hardening in the films. The highest hardness of $40.4 \pm 0.8 \mathrm{GPa}$ was obtained for 20 - $\AA$ TiN with 5- $\AA$-thick $\operatorname{SiN}(001)$ interlayers, compared to monolithic TiN at $20.2 \pm 0.9 \mathrm{GPa}$.
\end{abstract}

\section{INTRODUCTION}

Recent studies on $\mathrm{TiN}_{\mathrm{SiN}}$ multilayers have shown that SiN layers can grow epitaxially on TiN layers. ${ }^{1-4}$ This gives rise to strong interfacial bonding and effective superlattice hardening, with hardness values higher than for both the monolithic constituents [TiN and $\operatorname{SiN}_{x}(x \geqslant$ 1)] and multilayers containing thicker, amorphous $\operatorname{SiN}_{x}$ $\left(\mathrm{a}-\mathrm{SiN}_{x}\right)$ layers. ${ }^{1,4} \mathrm{TiN} / \mathrm{SiN}_{x}$ multilayers grown on oxidized $\mathrm{Si}$ substrates result in polycrystalline films, and for thin $(\sim 3 \AA) \mathrm{SiN}_{x}$ layers the typical columnar growth morphology for $\mathrm{TiN}$ is maintained. ${ }^{1,2}$ As the $\mathrm{SiN}_{x}$ layer thickness is increased, a- $\mathrm{SiN}_{x}$ starts to form, resulting in the growth of polycrystalline TiN layers composed of equiaxed grains. The highest reported hardness values for $\mathrm{TiN} / \mathrm{SiN}_{\mathrm{x}}$ multilayers are observed for $\mathrm{SiN}_{x}$ layer thicknesses on the order of 3-7 $\AA^{1,3,5}$ This thickness is comparable to the thickness of the amorphous tissue phase that has been assumed to surround TiN nanocrystallites within $\mathrm{TiN}_{-} \mathrm{SiN}_{x}$ nanocomposites at the percolation threshold $\left(10-20\right.$ vol\% $\left.\mathrm{Si}_{3} \mathrm{~N}_{4}\right){ }^{6-8}$ Here the maximum hardness is achieved at a Si content of 6-10 at.\%, corresponding to $\mathrm{SiN}_{x}$ tissue phase thickness of 1-2 mono-

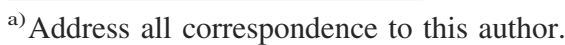

e-mail: soderberg.hans@gmail.com

DOI: $10.1557 / J M R .2007 .0412$
}

layers $(\sim 2-4 \AA) .{ }^{8}$ Outstanding questions for the TiN$\mathrm{SiN}_{x}$ system are for the nature of the $\mathrm{SiN}_{x}$ layers in terms of structure and nitrogen content as a function of layer thickness. For the crystalline $\mathrm{SiN}$ (c-SiN) tissue phase, the degree of coherency to the TiN phase also needs to be determined.

In the present work, epitaxial $\mathrm{TiN} / \mathrm{SiN}(001)$ superlattices and polycrystalline $\mathrm{TiN} / \mathrm{SiN}_{x}$ multilayer films have been grown by dual-reactive magnetron sputtering. The nearly identical lattice parameters for $\mathrm{MgO}$ and $\mathrm{TiN}$ (4.213 and $4.242 \AA$, respectively ${ }^{9,10}$ ) were used to obtain epitaxial single-crystal TiN films, as known from the literature. ${ }^{11}$ To address the topics above, our depositions of superlattices and multilayers were made over a wide range of deposition conditions, including different layer thicknesses, substrate temperatures, and substrate bias voltages during $\operatorname{SiN}_{x}$ deposition.

\section{EXPERIMENTAL PROCEDURES}

Film deposition was carried out in a reactive dualmagnetron direct current (dc) sputtering system ${ }^{12}$ utilizing computer-controlled shutters to achieve the layered structure. Targets consisted of pure titanium and silicon discs, with purities of $99.97 \%$ and $99.99 \%$, respectively. Argon and nitrogen were used as working and reactive gas at partial pressures of 4.0 and 0.5 mTorr, respectively. 
Purity of both gases was $99.99999 \%$. The base pressure in the chamber was always $<10^{-6}$ Torr before depositions commenced. The targets were run in a constant-current mode, fed with 0.8 and 0.2 A for the Ti and Si targets, respectively. Resulting deposition rates were $\sim 1.5 \AA / \mathrm{s}$ for TiN and $\sim 0.5 \AA / s$ for $\operatorname{SiN}_{x}$.

Single-crystal, (001)-oriented $\mathrm{MgO}(10 \times 10 \times$ $0.5 \mathrm{~mm}^{3}$ ) wafers were used as substrates. Before insertion in the deposition chamber, the substrates were ultrasonically cleaned in trichloroethylene, acetone, and isopropyl alcohol baths. Prior to deposition, the substrates were preheated in situ for $1 \mathrm{~h}$ at $>800{ }^{\circ} \mathrm{C}$. Using the described procedure, high-quality epitaxial TiN films have been grown as observed in low-energy electron diffraction $^{4}$ and reflection high-energy electron diffrac$\operatorname{tion}^{13}$ studies. This confirms the formation of a highquality $\mathrm{MgO}$ surface through annealing, which has previously been reported. ${ }^{14}$ In addition, the high temperature removes surface hydroxide and carboxide, a process that has been thoroughly studied in annealing experiments in air. ${ }^{15}$ The substrate temperature was controlled by a resistive heater positioned behind the substrate holder. All samples except those used for rate calibration were deposited with a $500-\AA$ TiN template layer at $700{ }^{\circ} \mathrm{C}$. After the template, 30 bilayers were deposited with varied deposition conditions. In addition, some samples were also grown to a total thickness of $0.5 \mu \mathrm{m}$ for more reliable nanoindentation experiments.

The primary set of samples consisted of multilayers grown at $500{ }^{\circ} \mathrm{C},-30 \mathrm{~V}$ bias, constant TiN layer thickness $(\sim 40 \AA)$, and varying $\mathrm{SiN}_{x}$ layer thickness (1.5$25 \AA$ ). In addition, at $500{ }^{\circ} \mathrm{C}$ and constant TiN and $\mathrm{SiN}_{x}$ layer thicknesses, 40 and $5 \AA$, respectively, bias was varied between floating potential $(\sim-17 \mathrm{~V})$ and $-60 \mathrm{~V}$. For the same layer thicknesses and a constant bias of $-30 \mathrm{~V}$, samples were also grown at 300,500 , and $700{ }^{\circ} \mathrm{C}$. With a constant $\mathrm{SiN}_{x}$ layer thickness of $5 \AA$, at $500{ }^{\circ} \mathrm{C}$, and $-30 \mathrm{~V}$ bias, the TiN layer thickness was varied (5, 20, and $40 \AA$ ). Finally, two multilayer samples were grown for dedicated elastic recoil detection analysis (ERDA), as described in Table I. Each sample was constituted of six multilayer TiN/SiN ${ }_{x}$ stacks. In the $\Omega$-series sample, the $\operatorname{SiN}_{x}$ layer thickness was kept low and the $\mathrm{SiN}_{x}$-to-TiN layer thickness ratio was kept constant, while the number of interfaces for each stack was varied. Each subsequent stack was grown to higher thickness to account for the lower ERDA depth resolution with increasing probing depths. In the $\Gamma$-series sample, the ratio of $\mathrm{SiN}_{x}$-to-TIN individual layers was varied, while keeping the total bilayer thickness constant.

$\mathrm{X}$-ray reflection (XRR) measurements were conducted to determine the multilayer periods $(\Lambda)$, i.e., bilayer thickness of the films, and also to confirm that the deposition rate calibrations were correct. The multilayer periods were calculated from the reflectivity peak positions
TABLE I. Design of $\mathrm{TiN} / \mathrm{SiN}_{x}$ multilayer samples for elastic recoil detection analysis; $\Omega$-series (left column) and $\Gamma$-series (right column).*

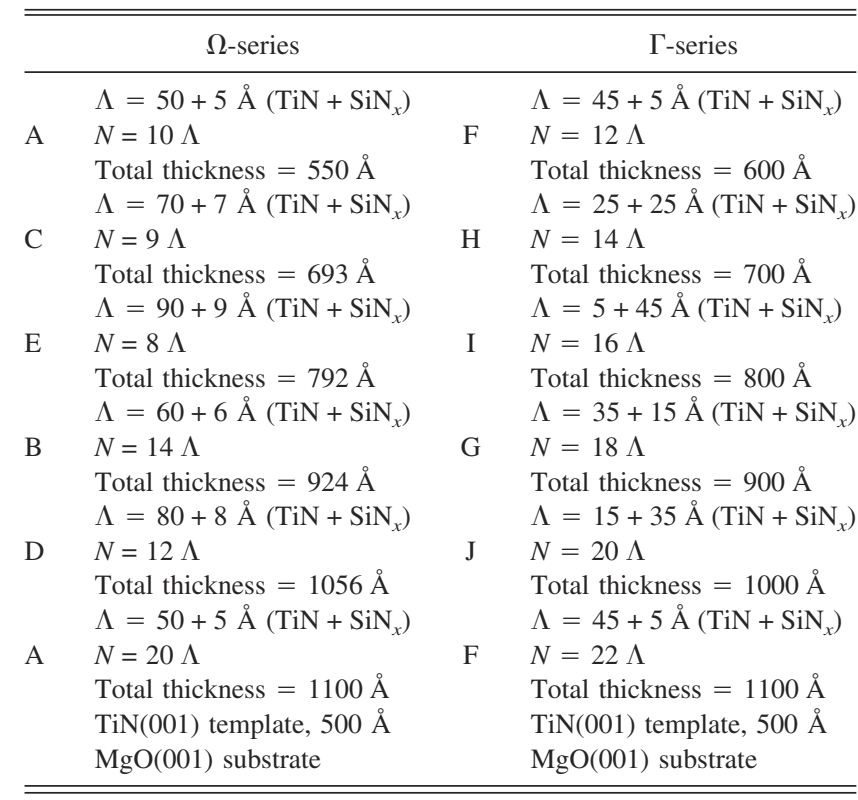

*A-J indicate different multilayer stacks within the respective sample, for which the first and last multilayer stack have the same configuration. $\Lambda$ equals the multilayer period, i.e., bilayer thickness. $N$ equals the number of bilayer repeats.

and a procedure described elsewhere. ${ }^{16}$ The XRR studies were conducted on a Philips PW1729 diffractometer with $\theta-2 \theta$ geometry and $\mathrm{Cu} \mathrm{K}_{\alpha}$ radiation. XRR simulations were carried out with the IMD extension ${ }^{17}$ under the XOP software. ${ }^{18}$ For high-resolution x-ray diffraction (HRXRD), we used a Philips four-axis materials research diffractometer (MRD) equipped with a primary monochromator utilizing a graded, parabolically bent multilayer mirror followed by a two-bounce $\mathrm{Ge}(220)$ monochromating crystal (producing $\mathrm{Cu} \mathrm{K}_{\alpha 1}$ radiation). Reciprocal space mapping was made using a $15^{\circ}$ asymmetrically cut two-bounce $\mathrm{Ge}(220)$ analyzer crystal.

Cross-sectional transmission electron microscopy (TEM) specimens were prepared by mechanical grinding and polishing. Low-angle Ar ion milling, in a Gatan PIPS ion miller, was used to obtain electron transparent specimens. TEM imaging was performed in a FEI Technai $\mathrm{G}^{2}$ UT FEG operated at $200 \mathrm{kV}$ for $1.9-\AA$ point resolution. For scanning transmission electron microscopy (STEM) imaging a FEI (Eindhoven, The Netherlands) Titan 80$300 \mathrm{~S} / \mathrm{TEM}$ microscope, operated at $300 \mathrm{kV}$ was used to achieve better than $1.4-\AA$ point resolution. A high-angle annular dark-field detector (HAADF) was used to obtain STEM images. A camera length of $300 \mathrm{~mm}$ was selected to provide sufficient contrast from the $\mathrm{SiN}_{x}$ layers.

Both ERDA samples were analyzed using a $347-\mathrm{MeV}$ $\mathrm{Au}$ ion beam. The resulting energy-over-depth recoil spectra were transformed to depth resolved concentration 
profiles using the KONZERD code. Additionally, all spectra were fitted with the SIMNRA computer code. Details on the experimental setup and the fitting procedure can be found elsewhere. ${ }^{19}$

The film hardness was evaluated with a Nanoindentor II instrument equipped with a diamond Berkovich indenter. Several different loads $(2-10 \mathrm{mN})$ were used, and for each load at least 10 indents were made. The loading cycle consisted of two loadings to maximum load $\left(L_{\max }\right)$ with an intermediate unloading to $10 \% L_{\max }$. Hold periods were applied at the intermediate $10 \% L_{\max }$ load to correct for any thermal drift and at the final $L_{\max }$ to reveal the presence of any creep. Hardness was obtained through the procedure developed by Oliver and Pharr. ${ }^{20}$ From the indentation data generated for loads not influenced by the substrate, an average hardness value and its standard deviation were calculated.

\section{RESULTS}

All as-deposited films exhibited a number of peaks in XRR scans confirming a layered structure with a strong compositional modulation. The majority of samples thus showed two to four sharp reflectivity peaks and Kiessig fringes. Even for a $\mathrm{SiN}_{x}$ interlayer thickness as thin as $\sim 1.5 \AA$, a multilayer peak was observed, though weak. Figure 1 shows the reflectivity curve with corresponding simulated curve from a sample grown at $500{ }^{\circ} \mathrm{C}$. The sample was grown without a TiN template layer and with $\mathrm{TiN}$ and $\mathrm{SiN}_{x}$ layers of approximately equal thickness, $11 \AA$; hence the low intensity of the second Bragg reflection. The simulated ${ }^{17,18}$ curve matches the two peaks, and the resulting wavelength value is close to the expected one based on the deposition rate calibrations. Good agreement with the majority of the Kiessig fringes

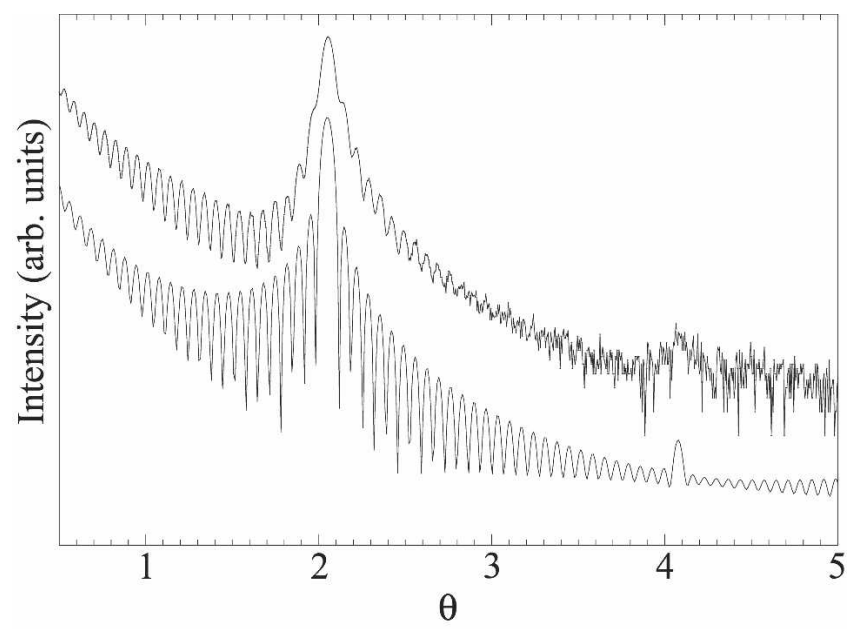

FIG. 1. Measured (upper) and simulated (lower) x-ray reflectivity curves from a $\mathrm{TiN} / \mathrm{SiN}_{x}$ multilayer film grown on $\mathrm{MgO}(001)$ at $500{ }^{\circ} \mathrm{C}$. TiN and $\mathrm{SiN}_{x}$ layer thicknesses are $\sim 11 \AA$. Curves are vertically separated for clarity and plotted against $\theta$. is also observed, resulting in an interfacial thickness of $1 \AA$, defined as the root-mean-square interfacial roughness and the interfacial width. ${ }^{17}$

Figure 2 shows the HRXRD diffractograms from the set of samples deposited at $500{ }^{\circ} \mathrm{C}$ with varying $\mathrm{SiN}_{x}$ layer thicknesses and constant TiN layer thickness ( $\sim 40 \AA$ ). Also added is the diffractogram from the monolithic TiN film which exhibits two sharp peaks, corresponding to TiN 002 and MgO 002. With the introduction of $\mathrm{SiN}_{x}$ interlayers, already at thicknesses of $\sim 2 \AA$ the first indications of a first-order superlattice (SL) peak are observed around $40^{\circ} 2 \theta$. As the $\mathrm{SiN}_{x}$ interlayer thickness increases, the first-order SL peak becomes more distinct. Simultaneously, second-order SL peaks at lower $2 \theta$ angles are also observed. The formation of SL peaks is evidence of the growth of a highly repetitive layered structure with flat interfaces. In addition to the SL peaks, from a $\mathrm{SiN}_{x}$ thickness of $2.6 \AA$, low-intensity fringes of two different modulation periods are observed, the longer of these periods is marked with $\Delta 2 \theta$ in the figure. For thicker $\mathrm{SiN}_{x}$ layers, only the longer fringe period is preserved. These fringes are so-called Pendellösung fringes,${ }^{21}$ or finite-thickness fringes, originating from interference between the different sections within the film (i.e., multilayer, template layer, substrate) and their interfaces. The TiN 002 peak changes appearance with increasing $\mathrm{SiN}_{x}$ layer thicknesses. It changes from a very sharp, for monolithic TiN and for multilayers containing thin $\mathrm{SiN}_{\mathrm{x}}$ interlayers, to a broad appearance as the $\mathrm{SiN}_{x}$ layers approach $13 \AA$. In addition, a small shift toward higher $2 \theta$ values of the TiN 002 maximum intensity position occurs for samples with $\mathrm{SiN}_{x}$ layers of 2.6-5.5 thickness.

XRR and XRD curves from samples with varying

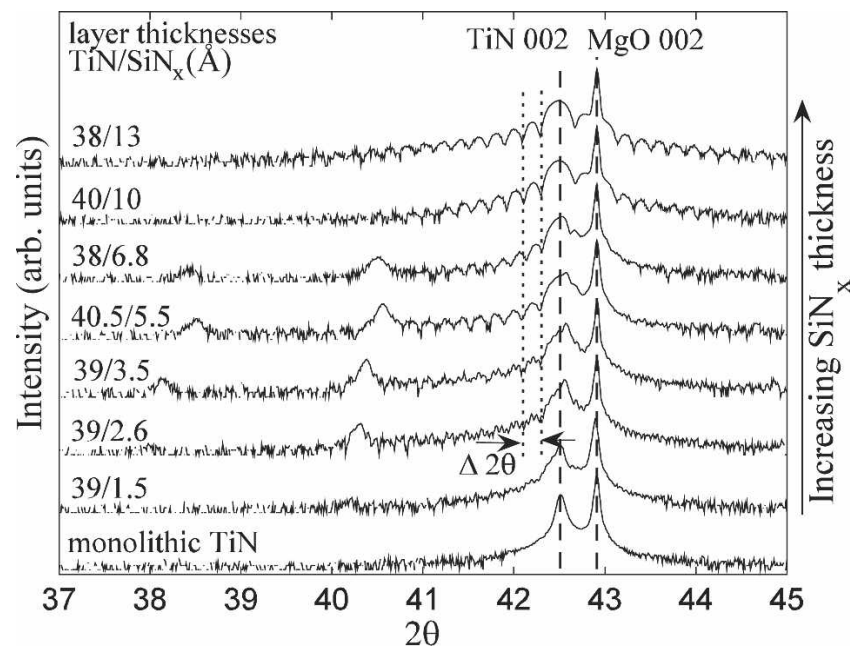

FIG. 2. HRXRD scans from a monolithic TiN(001) film and a series of TiN/SiN $x$ multilayers with increasing $\mathrm{SiN}_{x}$ layer thickness grown on $\mathrm{MgO}(001)$ at $500{ }^{\circ} \mathrm{C}$. The interference fringe width $(\Delta 2 \theta)$ is marked and corresponds to the template layer thickness (see Sec. IV). 
negative substrate bias, $V_{\mathrm{s}}$, showed that $V_{\mathrm{s}}=30-40 \mathrm{~V}$ produces the most distinct reflection and diffraction curves. However, only a slight degradation was seen for samples grown at floating potential (approximately $-17 \mathrm{~V}$ ) and $V_{\mathrm{s}}=50-60 \mathrm{~V}$. Figure 3 shows XRR curves from TiN/SiN ${ }_{x}$ multilayer grown at $T_{\mathrm{s}}=300,500$, and $700{ }^{\circ} \mathrm{C}$. All samples exhibit reflection peaks and intermediate Kiessig fringes, supporting the growth of a compositional modulation in the three cases. For the sample grown at $300{ }^{\circ} \mathrm{C}$, the XRD curve display only SL peaks, suggesting lower interfacial quality. This is likely an effect of the reduced ad-atom mobility, which promotes amorphous phase formation. For $T_{\mathrm{s}}=500{ }^{\circ} \mathrm{C}$, both SL peaks and fringes are observed, indicating a film with higher crystalline order than for the $300{ }^{\circ} \mathrm{C}$ case. For $T_{\mathrm{s}}=700{ }^{\circ} \mathrm{C}$, peak broadening of TiN 002 occurs and XRR shows that both SL peaks and fringes, however less distinct, compare to the $500{ }^{\circ} \mathrm{C}$ case. These observations are consistent with the formation of a-SiN ${ }_{x}$ layers with low roughness, suggesting a lower stability for the c-SiN at 700 than at $500{ }^{\circ} \mathrm{C}$.

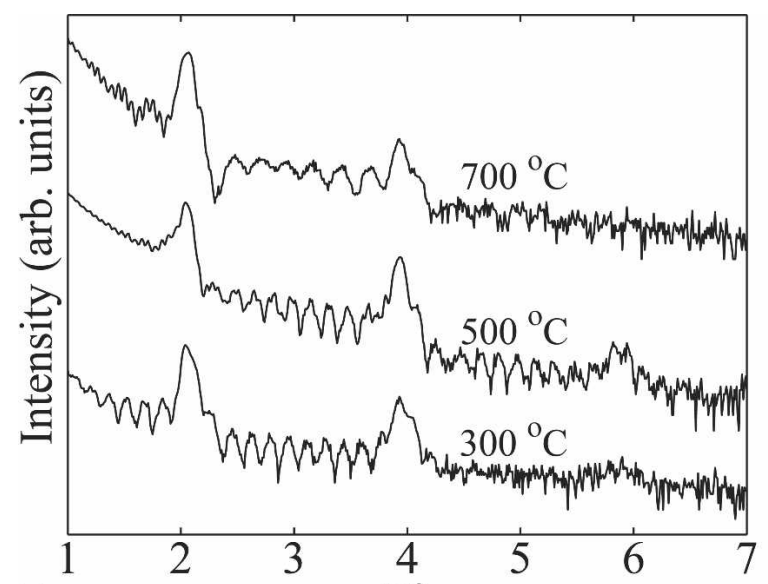

(a) $2 \theta$

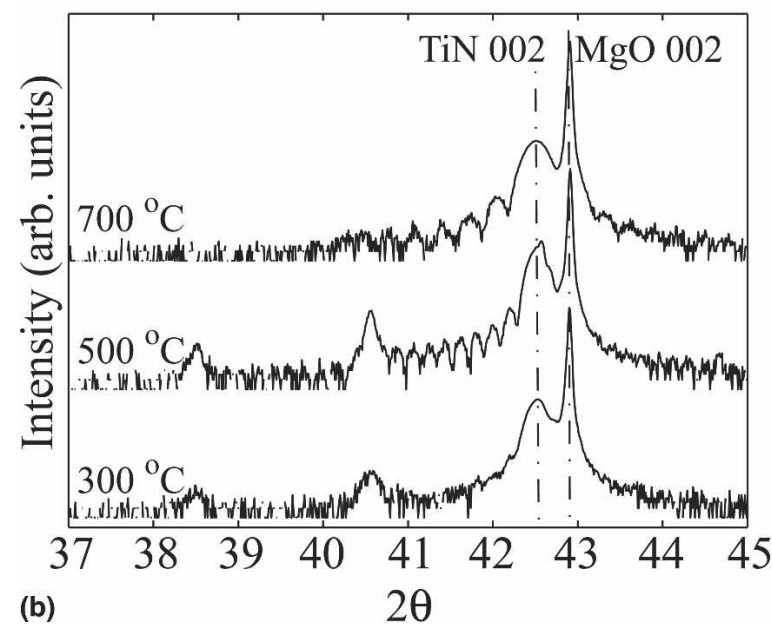

FIG. 3. (a) X-ray reflection and (b) diffraction scans for $\mathrm{TiN} / \mathrm{SiN}_{x}$ multilayers grown at 300,500 , and $700{ }^{\circ} \mathrm{C}$. Layer thicknesses are 40 and $5 \AA$ for $\mathrm{TiN}$ and $\mathrm{SiN}$, respectively.
Figures 4(a) and 4(b) show the reciprocal-space maps (RSM) around the 002 and $113 \mathrm{MgO}$ substrate peaks, respectively, from a superlattice film grown at $500{ }^{\circ} \mathrm{C}$ with $\mathrm{TiN}$ and $\mathrm{SiN}_{x}$ layer thicknesses of 40 and $5.5 \AA$, respectively. All observed intensity is well confined in reciprocal space, and no indication of any polycrystalline growth exists. In addition to the $\mathrm{MgO} 002$ and TiN 002 primary peaks, SL peaks, Pendellösung fringes (P), and analyzer streaks (A) are also observed in the two RSMs. The RSM results indicate the growth of well-defined single-crystal films, i.e., superlattices. To further check the symmetry of the film in Fig. 4 (which shows one of the four 113 regions), $360^{\circ} \phi$-scans over the asymmetric TiN 113 peak were performed. They revealed only four peaks symmetrically spaced, which is indicative of a single crystal, in our case, a TiN/SiN(001) superlattice.

Figures 5(a) and 5(b) show high-resolution TEM micrographs from $\mathrm{TiN} / \mathrm{SiN}_{x}$ multilayers with $5.5-\AA$ $\mathrm{SiN}(001)$ layers and $13-\AA \mathrm{SiN}_{x}$ layers, respectively. In Fig. 5(a), the crystalline TiN lattice is maintained from one layer to the next via the epitaxially stabilized SiN layers. These can be seen as brighter lines of contrast, apparently without an amorphous component. This observation confirms the single-crystal nature of the superlattice sample. The difference in contrast derives from the incoherent scattering strength of the present atoms and absorption effects. Interface strain is visible at the $\mathrm{TiN} / \mathrm{SiN}(001)$ interfaces. It is suggested that the layers
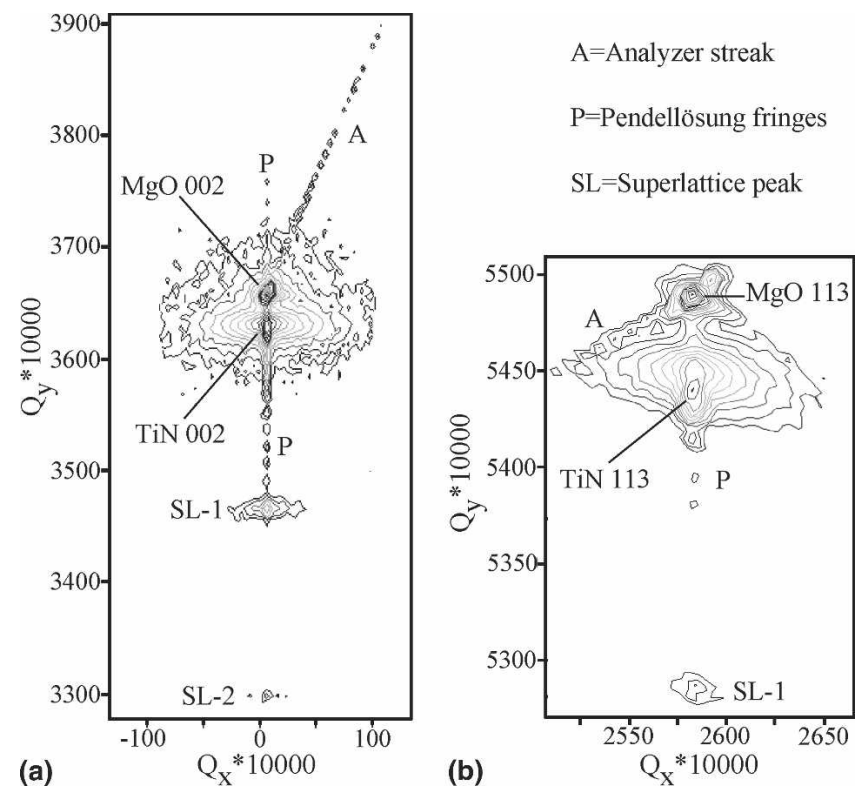

FIG. 4. Reciprocal-space maps from a TiN/SiN(001) superlattice film with 5.5- $\AA$ thick SiN layers around the region of (a) the 002 orientation and (b) the 113 orientation. Superlattice peaks (SL), Pendellösung (P), and analyzer (A) streaks are marked. Axis units are reciprocal lattice units normalized with respect to the diameter of the Ewald sphere, $k_{0}=2(2 \pi / \lambda)$, i.e., the theoretical maximum distance from the origin for a specific wavelength. 


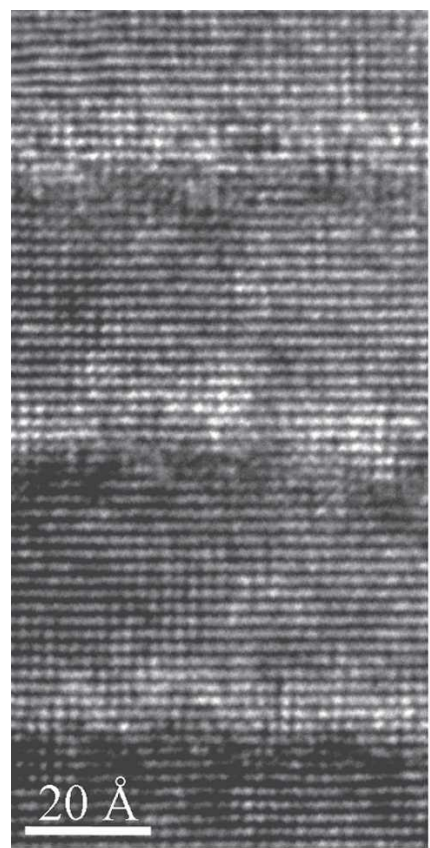

(a)

(b)

FIG. 5. Cross-sectional TEM micrographs from TiN/SiN ${ }_{x}$ multilayers containing (a) $5.5-\AA \mathrm{AiN}(001)$ and (b) $13-\AA \mathrm{SiN}_{x}$ layers, respectively. TiN layer thicknesses are $\sim 40 \AA$.

are strained to accommodate for the epitaxial growth of under stoichiometric (with respect to $\mathrm{Si}_{3} \mathrm{~N}_{4}$ ) $\mathrm{SiN}_{x}$.

For a $\mathrm{SiN}_{x}$ layer thickness of $13 \AA$ [Fig. 5(b)], amorphous interlayers begin to form, which results in the loss of the single-crystal template layer for each new TiN layer, see Fig. 2 where no SL peaks are observed at this $\mathrm{SiN}_{x}$ layer thickness. Consequently, a more or less polycrystalline film results, similar to earlier growth studies of TiN/SiN ${ }_{x}$ multilayers made on oxidized Si wafers. ${ }^{1}$ As shown in Fig. 5(b), at $13 \AA \mathrm{SiN}_{x}$, the layers grow amorphous. However, the initial atomic layers in each $\mathrm{SiN}_{x}$ layer exhibit epitaxial growth, and the transition to polycrystalline/amorphous phase occurs gradually with increasing $\mathrm{SiN}_{x}$ thickness.

STEM micrographs obtained from the layers next to the $\operatorname{TiN}(001)$ template layer are shown in Fig. 6. The overview image in Fig. 6(a) reveals that the first few TiN and SiN layers grow epitaxially on each other. Epitaxial growth was seen over the first five TiN layers with decreasing intensity. In the fifth layer, the rightmost grain assumes a completely different orientation, revealing the onset of TiN renucleation which gives rise to a mixed epitaxial/polycrystalline film nature. An atomicresolution STEM image shows the first $\mathrm{SiN}(001)$ layer [Fig. 6(b)]. Because of the difference in atomic mass $(Z)$ and the contribution from mass contrast $\left(Z^{2}\right)$, the $\mathrm{SiN}$ layer contrast is weak. Nevertheless, the epitaxial nature of the SiN layer is indisputable. A misfit dislocation in the top of the layer is identified, supporting the TEM

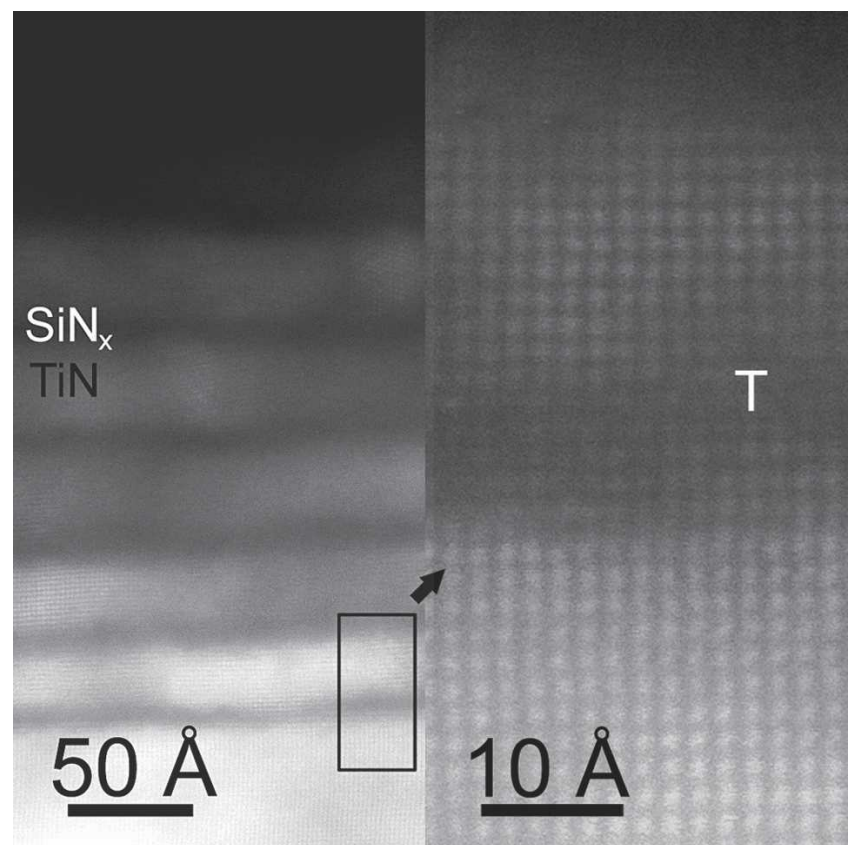

(a)

(b)

FIG. 6. Atomic resolution STEM images from a TiN/SiN $\mathrm{S}_{x}$ multilayer film with $13-\AA$ thick $\operatorname{SiN}_{x}$ layers in (a) overview showing also the $\mathrm{TiN}(001)$ template layer (bottom), and (b) atomic resolution of a region with epitaxial growth marked in (a) containing also a misfit dislocation (denoted by the white " $T$ ").

observations and the assumption of a small lattice mismatch between TiN and c-SiN.

Figure 7 shows the ERDA concentration profiles as derived from the KONZERD code. The $\Omega$-series depicted in Fig. 7(a) exhibits a low overall silicon content and a more or less crystalline structure; it also shows oxygen and other impurity contaminations around and below $0.1 \%$, independent of the number of interfaces. The nitrogen concentration in all six stacks of the multilayer sample was $\sim 50 \% \pm 1 \%$, which corroborates our previous assignment of stoichiometric $\mathrm{SiN}$ in its strainstabilized cubic polytype. In contrast, for the $\Gamma$-series with sections containing c-SiN and a-SiN ${ }_{x}$, an increase in silicon concentration is clearly correlated with a nitrogen increase, as shown in Fig. 7(b). Also, the nitrogen concentration lies considerably above 50\% $(x>1)$, pointing toward formation of amorphous $\mathrm{Si}_{3} \mathrm{~N}_{4}$. In addition, argon shows a strong peaking for highest silicon contents, possibly due to easier argon incorporation from the sputtering gas into an amorphous $\mathrm{SiN}_{x}$ matrix of lower density compared to c-SiN and TiN. We assume that the amorphous $\mathrm{SiN}_{x}$ phase has the composition $\mathrm{Si}_{3} \mathrm{~N}_{4}$ since growth was carried out in a highly $\mathrm{N}_{2}$-rich ambient. This assumption was checked by the growth, under the same conditions, of a 500-nm-thick $\mathrm{SiN}_{x}$ layer where the composition was subsequently determined by ERDA to be $\mathrm{Si}_{3} \mathrm{~N}_{4}$. 


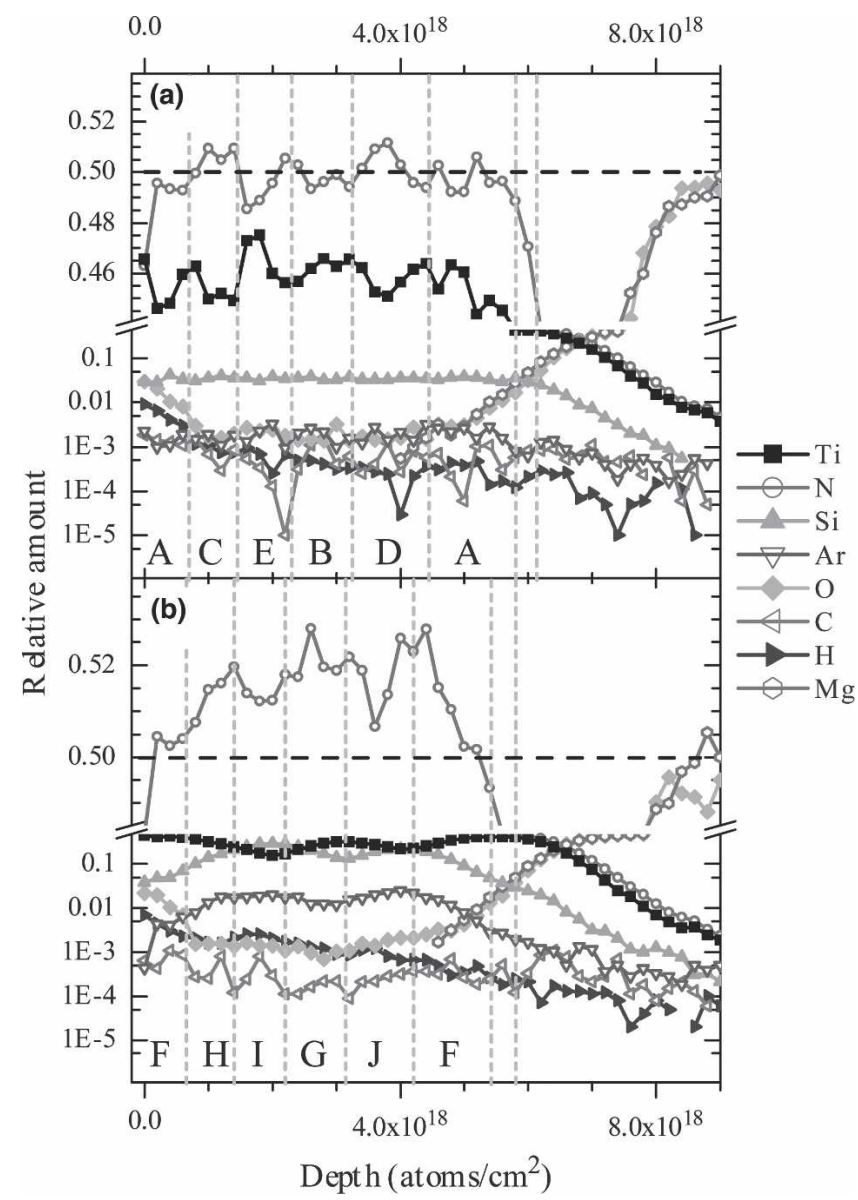

FIG. 7. Elastic recoil detection analysis concentration depth profiles calculated by the KONZERD code. Dashed vertical bars indicate the six stacks of each sample, as defined in Table I. In (a) the $\Omega$-series, with a constant $\mathrm{TiN} / \mathrm{SiN}_{x}$ ratio, varying the number of interfaces, and keeping a low $\mathrm{SiN}_{x}$ concentration, and (b) the $\Gamma$-series with a constant bilayer thickness and varying the $\mathrm{SiN}_{x}$ content.
Table II shows the quantified ERDA results from both samples as obtained from fitting the experimental spectra by SIMNRA. The alternating $\mathrm{TiN} / \mathrm{SiN}_{x}$ single layers were disregarded in the analysis; instead, a homogeneous mixture of $\mathrm{Ti}$ and $\mathrm{Si}$ within each of the six-layer stacks was assumed. The table shows the average values for each stack. It is notable that for the $\Omega$-series, the impurity-oxygen, carbon, hydrogen, and argon-levels are constant over the whole thickness while the number of $\mathrm{TiN}_{-} \mathrm{SiN}_{x}$ interfaces is increased from 8 to 20. Segregation of impurities toward these interfaces can hence be excluded. For the $\Gamma$-series, as already seen from the concentration profiles in Fig. 7, the nitrogen concentration increases with $\mathrm{SiN}_{x}$ content. However, an exact calculation of $x$ in the $\operatorname{SiN}_{x}$ is hindered by the up to $2 \% \mathrm{Ar}$ incorporation that is assumed to be incorporated in the a-SiN ${ }_{x}$ layers, which are of lower density than TiN.

In Fig. 8(a), the hardnesses from TiN/SiN ${ }_{x}$ multilayer samples with varying $\mathrm{SiN}_{\mathrm{x}}$ layer thickness are shown. These films are grown with $\sim 40 \AA \mathrm{TiN}$, at $500{ }^{\circ} \mathrm{C}$, and with a bias of $-30 \mathrm{~V}$. For comparison, hardness data on polycrystalline multilayers deposited on oxidized $\mathrm{Si}$ wafers from our earlier work ${ }^{1}$ are also added. In this study, as well as in the previous study, ${ }^{1}$ the hardness is higher for the multilayers compared to the monolithic films. This is in agreement with the large number of reports throughout the last two decades concerning various superlattice films with strong compositional modulation for coherently strained layers of phases with different shear moduli. ${ }^{22-24}$ In the set of samples grown in this study, the highest hardness is observed for a $\mathrm{SiN}_{x}$ layer thickness of $7.5 \AA$, reaching $34.0 \pm 2.4 \mathrm{GPa}$. With the formation of a-SiN $x$ and the growth of polycrystalline TiN layers in a "nanobrick-like"1 structure, the hardness drops to some

TABLE II. Fitted elastic recoil detection analysis depth profile concentrations as calculated by SIMNRA.

\begin{tabular}{|c|c|c|c|c|c|c|c|c|c|c|}
\hline Sample & $\begin{array}{c}\text { Thickness } \\
\left(10^{15} \text { at. } / \mathrm{cm}^{2}\right)\end{array}$ & $\begin{array}{c}\mathrm{Ti} \\
(\AA)\end{array}$ & $\begin{array}{c}\mathrm{Ti} \\
\text { (at.\%) }\end{array}$ & $\begin{array}{c}\mathrm{Si} \\
(\AA)\end{array}$ & $\begin{array}{c}\mathrm{Si} \\
\text { (at.\%) }\end{array}$ & $\begin{array}{c}\mathrm{N} \\
\text { (at.\%) }\end{array}$ & $\begin{array}{c}\mathrm{H} \\
\text { (at.\%) }\end{array}$ & $\begin{array}{c}\mathrm{C} \\
\text { (at.\%) }\end{array}$ & $\begin{array}{c}\mathrm{O} \\
\text { (at. } \%)\end{array}$ & $\begin{array}{c}\mathrm{Ar} \\
\text { (at.\%) }\end{array}$ \\
\hline \multirow[t]{8}{*}{$\Omega$-series } & 15 & 0 & 24 & 0 & $\ldots$ & $\ldots$ & 23 & 5 & 48 & $\ldots$ \\
\hline & 700 & 50 & 46 & 5 & 3.5 & 50.08 & 0.05 & 0.05 & 0.1 & 0.2 \\
\hline & 750 & 70 & 46 & 7 & 3.5 & 50.03 & 0.05 & 0.05 & 0.15 & 0.2 \\
\hline & 850 & 90 & 45.85 & 9 & 3.5 & 50.25 & 0.05 & 0.05 & 0.1 & 0.2 \\
\hline & 950 & 60 & 45.62 & 6 & 3.5 & 50.5 & 0.05 & 0.05 & 0.1 & 0.2 \\
\hline & 1200 & 80 & 44.8 & 8 & 3.6 & 51.11 & 0.05 & 0.05 & 0.15 & 0.1 \\
\hline & 1350 & 50 & 44.5 & 5 & 3.6 & 51.44 & 0.05 & 0.05 & 0.15 & 0.2 \\
\hline & 330 & 500 & 50 & 0 & $\ldots$ & 49.95 & $\ldots$ & 0.05 & $\ldots$ & $\ldots$ \\
\hline \multirow[t]{8}{*}{$\Gamma$-series } & 15 & 0 & 25 & 0 & $\ldots$ & $\ldots$ & 20 & 5 & 50 & $\ldots$ \\
\hline & 650 & 45 & 44.59 & 5 & 5 & 50.015 & 0.025 & 0.05 & 0.1 & 0.22 \\
\hline & 750 & 25 & 26.24 & 25 & 19.2 & 52.11 & 0.25 & 0.05 & 0.15 & 2.0 \\
\hline & 800 & 5 & 12.2 & 45 & 32.89 & 52.46 & 0.3 & 0.05 & 0.1 & 2.0 \\
\hline & 950 & 35 & 36.3 & 15 & 11.1 & 51.555 & 0.025 & 0.2 & 0.1 & 0.9 \\
\hline & 1050 & 15 & 18.2 & 35 & 24.4 & 54.01 & 0.15 & 0.05 & 0.19 & 3.0 \\
\hline & 1300 & 45 & 44 & 5 & 4 & 51.456 & 0.025 & 0.02 & 0.19 & 0.3 \\
\hline & 350 & 500 & 50 & 0 & $\ldots$ & 49.95 & $\ldots$ & 0.05 & $\ldots$ & $\ldots$ \\
\hline
\end{tabular}



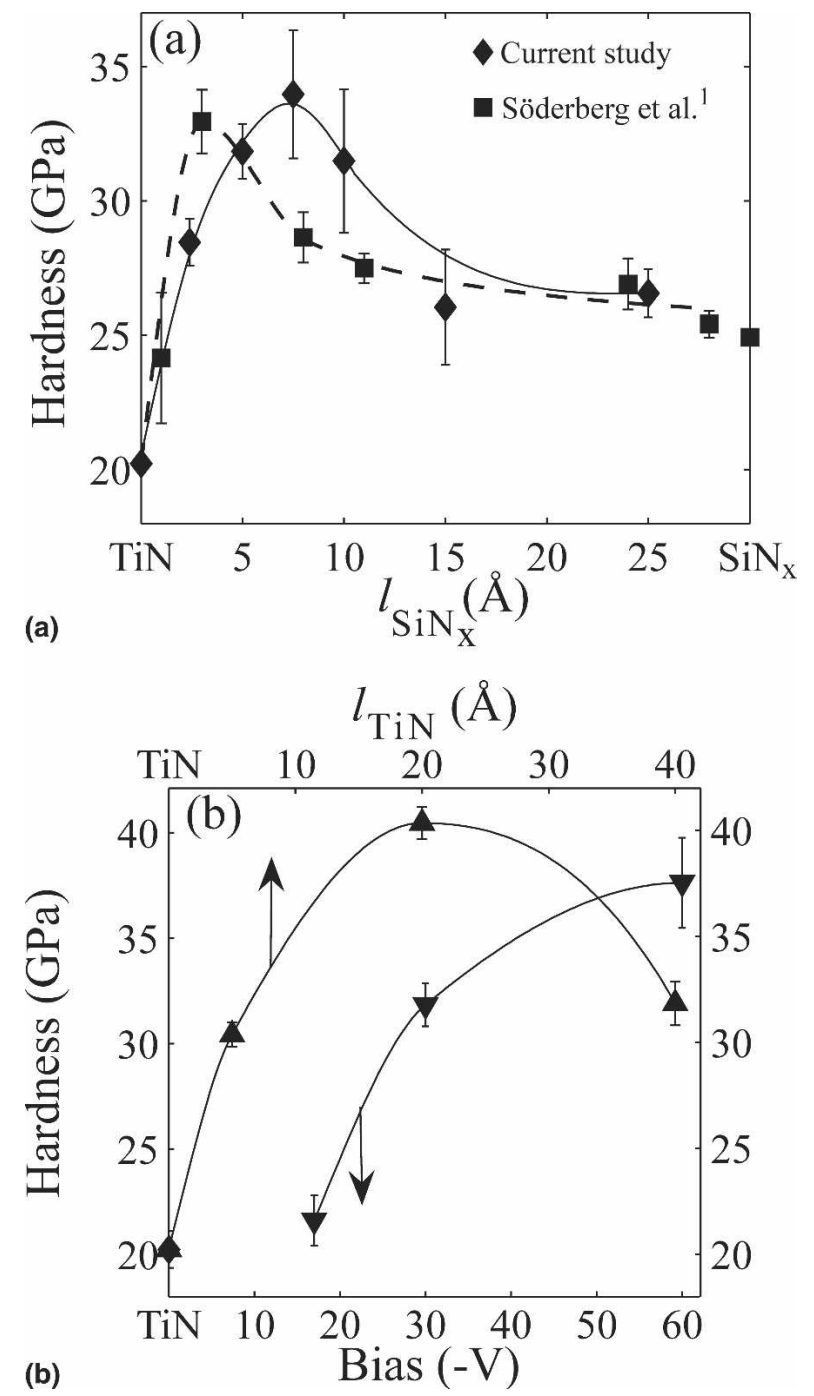

FIG. 8. (a) Nanoindentation hardness of $\mathrm{TiN} / \mathrm{SiN}(001)$ superlattice films grown on $\mathrm{MgO}$ (marked with diamonds) compared with polycrystalline $\mathrm{TiN} / \mathrm{SiN}_{x}$ multilayer samples grown on $\mathrm{Si}$ (see Ref. 1) (squares) as a function of $\mathrm{SiN}_{x}$ layer thickness. (b) Hardness as a function of TiN layer thickness (top $x$ axis) with $\mathrm{SiN}_{x}$ thickness constant at $5 \AA$, and hardness as a function of negative substrate bias, $V_{\mathrm{s}}$ (lower $x$ axis) with constant TiN and $\operatorname{SiN}_{x}$ thickness of 40 and $5 \AA$, respectively. Monolithic TiN was grown at $V_{\mathrm{s}}=30 \mathrm{~V}$.

extent and approaches that of monolithic $\operatorname{SiN}_{x}$. The monolithic single-crystal TiN film reached a hardness of $20.2 \pm 0.9 \mathrm{GPa}$, which is in accordance with previous studies $(20.3 \pm 0.8 \mathrm{GPa}){ }^{11}$

Similar to earlier results, ${ }^{25}$ the load-displacement curves indicate a higher elastic recovery for multilayer films compared to the TiN monoliths; e.g., for a $5-\mathrm{mN}$ load, the monolithic TiN film exhibited an elastic recovery of $\sim 33 \%$ whereas the corresponding values for multilayer films and monolithic $\mathrm{SiN}_{x}$ are higher, 50-60\%. As can be seen in Fig. 8(b), bias affects the hardness quite substantially. With floating potential (approximately $-17 \mathrm{~V}$ ) during $\mathrm{SiN}_{x}$ deposition the multilayer is only slightly harder than the monolithic TiN film grown at $-30 \mathrm{~V}$. Clearly, an increase in bias causes an increase in hardness, reaching a maximum of $37.6 \pm 2.1 \mathrm{GPa}$ at $-60 \mathrm{~V}$. In addition, changing the TiN layer thickness while maintaining the other parameters constant $\left(5-\AA \mathrm{SiN}, 500{ }^{\circ} \mathrm{C}\right.$, and $-30 \mathrm{~V}$ bias) made further tuning of the hardness possible. This resulted in a maximum hardness of $40.4 \pm 0.8 \mathrm{GPa}$ for $20 \AA$ TiN layers [Fig. 8(b)]. For 5- $\AA$ TiN, the hardness drops but is still well above the value for monolithic TiN film. Hardnesses in films grown with $40 \AA$ TiN and $5 \AA \mathrm{SiN}_{x}$ show similar hardness values when grown at 300 and $500{ }^{\circ} \mathrm{C}$ and at $32.1 \pm 3.4$ and $31.8 \pm 1.0 \mathrm{GPa}$, respectively. Increasing the deposition temperature to $700{ }^{\circ} \mathrm{C}$ results in an increase in hardness to $38.1 \pm 3.0 \mathrm{GPa}$.

\section{DISCUSSION}

The finding of this study offers new insights to the nature of $\mathrm{TiN} / \mathrm{SiN}_{x}$ interfaces. Based on the XRR and $\mathrm{XRD}$ results, TiN/SiN $x$ multilayer and $\mathrm{TiN} / \mathrm{SiN}_{x}(001)$ superlattice films are shown to have well-defined interfaces and layers. In the multilayers are polycrystalline $\mathrm{TiN}$ and a-SiN $x(x \geqslant 1)$ layers. In the superlattices, TiN and $\mathrm{SiN}$ layers are both stoichiometric and epitaxial. The reflectivity curves from layered films, of which one example is shown in Fig. 1, exhibit sharp reflectivity peaks with additional Kiessig fringes, suggesting flat and sharp interfaces, which is expected when the strong segregation tendency between $\mathrm{TiN}$ and $\mathrm{SiN}_{x}$ is considered. ${ }^{26,27} \mathrm{In}$ addition, the surface roughness can be maintained relatively low by using a low bias and well-polished substrates. The XRR simulations support this, suggesting an interfacial width in the low-ångström range.

The evidence for a strong compositional modulation within the films up to a SiN layer thickness of $6.8 \AA$ is apparent in Fig. 2, where the diffractograms display SL peaks and additional Pendellösung fringes between SL peaks and Bragg peaks. Due to the template layer, the resulting fringe pattern is complex for small SiN layer thicknesses due to superpositioning of interference from the superlattice and template layer. However, with increasing $\mathrm{SiN}_{x}$ layer thickness, the fringes get better defined, which is clearly seen by comparing, for example, the multilayers with 3.5- and $13-\AA \mathrm{SiN}_{x}$. The explanation for this behavior, and also for the loss of SL peaks for multilayers containing 10- to $13-\AA \mathrm{SiN}_{x}$, lies in the formation of a- $\mathrm{SiN}_{x}$. This formation is not occurring homogenously along the entire surface [see Figs. 5(b) and 6(a)], but instead, a more plausible development is that a-SiN ${ }_{x}$ forms locally on the growing c-SiN layer surface during deposition as the layer thickness is increased. The parallel growth of a polycrystalline and epitaxial TiN on a-SiN ${ }_{x}$ and c-SiN, respectively, evolve rougher interfaces [Fig. 5(b)] compared to the case with a completely epitaxial film. This prevents constructive interference, 
leading to the loss of the SL peaks and consequently Pendellösung fringes associated with the superlattice. However, the template layer is always the same, $500 \AA$ of epitaxial TiN. Here, interference between the multilayer (SL)/template layer interface and the template layer/ substrate interface cause fringes to appear in the diffractogram. This is also supported by a direct calculation of the thickness responsible for the interference fringes with a separation of $\Delta 2 \theta$ (Fig. 2) using Eq. (1),

$$
D=\frac{(m-n) \lambda}{2\left(\sin \theta_{\mathrm{m}}-\sin \theta_{\mathrm{n}}\right)},
$$

where $D$ equals the unknown thickness of the structure responsible for the measured fringes, $\theta_{\mathrm{m}}$ and $\theta_{\mathrm{n}}$ are the angular position for fringe $\mathrm{m}$ and $\mathrm{n}$, respectively, and $\lambda=1.54056 \AA$. A calculation of $D$ for the different samples in Fig. 2 results in $500 \AA$, i.e., the template layers' thickness. Hence, Pendellösung fringes originate from interference events within the films due to the high crystal quality of the superlattices and template layers. These types of fringes are only observed from layers with very high crystal quality, such as non-relaxed semiconductor epilayers, e.g., $\mathrm{Al}_{x} \mathrm{Ga}_{1-x} \mathrm{As} / \mathrm{GaAs}, \mathrm{Ge}_{1-y} \mathrm{C}_{y} /$ $\mathrm{Ge}_{1-x-y} \mathrm{Si}_{x} \mathrm{C}_{y}, \mathrm{Mn} / \mathrm{GaAs}$, and $\mathrm{MnGa} / \mathrm{GaAs} .{ }^{28-30}$

The high crystal quality indicated by XRR and XRD is confirmed by RSM (Fig. 4) and (S)TEM [Figs. 5(a) and 6(a)], where the RSMs display SL peaks and extra Pendellösung fringes, and in the STEM [Fig. 6(b)], the stabilization of c-SiN is evident. No signs of any polycrystalline growth are observed as long as the SiN thickness is kept around this thickness $(5.5 \AA)$ or lower. The observed c-SiN lattice matches the TiN lattice well [Figs. 5(a) and 6(b)]. This agrees with recent density-functional theory calculations on $\mathrm{NaCl}$ structure $\mathrm{SiN}$ and $\mathrm{TiN}$ revealing lattice parameters for $\mathrm{NaCl}-\mathrm{SiN}$ and $\mathrm{NaCl}-\mathrm{TiN}$ equal to $0.99 a_{0}$ and $0.986 a_{0}$, respectively, ${ }^{2,27}$ where $a_{0}$ is a TiN lattice parameter. ${ }^{10}$ This difference in lattice parameter between the two phases is sufficient to be detected with XRD; however, no SiN peaks are observed, suggesting that the two phases are coherently strained to each other. Specifically, the small shift of the TiN 002 peak toward higher $2 \theta$ angles for samples containing 2.6- to 5.5- $\AA \mathrm{SiN}$ (see Fig. 2) is indicative of a strain within the films, yielding an average superlattice 002 plane spacing that is smaller than that of pure TiN. This strain has likely additional sources, including thermal mismatch between film and substrate, thermal mismatch between the interlayers within the film, and point defects generated in the collision cascade during growth. It is apparent that the coherency strain contribution is the dominant one since the HRXRD peak shift disappears with the loss of epitaxial growth at $\sim 7$ - to $13-\AA \mathrm{SiN}_{x}$.

As discussed by Söderberg et al., ${ }^{2}$ the stabilization of metastable c-SiN can be attributed to pseudomorphic forces, i.e., a minimization of the systems' interfacial energy by the formation of a coherent interface and lowering of the strain energy during nucleation and coalescence. As the metastable layer grows thicker, the elastic strain energy and molar enthalpy increase until the crystalline growth breaks down by $\mathrm{a}-\mathrm{SiN}_{\mathrm{x}}$ formation. For this process, it was further argued ${ }^{4}$ that a transition in bonding coordination in $\mathrm{SiN}_{x}$ from 6- to 4-folded follows, i.e., from octahedral to tetrahedral. Thus, the $\mathrm{N}$ content $(x)$ should increase from $x \approx 1$ toward $1.33\left(\right.$ compare $\left.\mathrm{Si}_{3} \mathrm{~N}_{4}\right)$. The ERDA results in Fig. 7 and Table II support an

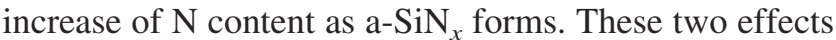
are responsible for the rapid transition to the amorphous phase, compared to epitaxial stabilization in other metastable material systems, e.g., AlN, where the stoichiometry remains constant. ${ }^{31}$

The multilayers show the highest hardness for thinner $\mathrm{SiN}_{x}$ layer thicknesses, Fig. 8(a). Within a single-crystal film, no grain boundaries exist that can act as dislocation obstacles. Instead, dislocation hindrance is obtained through Koehler hardening ${ }^{32}$ and from coherency strains due to the strained epitaxial $\mathrm{SiN}(001)$ interlayers. The grain boundaries formed in polycrystalline films with columnar epitaxial layer growth ${ }^{1,2}$ provides additional dislocation obstruction. On the other hand, it has been shown that columnar grain boundaries are sites where a material tends to shear and glide during deformation. ${ }^{16,33,34}$ The highest hardness observed in Fig. 8(a) for a film grown on $\mathrm{MgO}$ is $\sim 7-\AA \mathrm{SiN}$, to be compared to the polycrystalline case where reported highest hardness values are around 3- to $7-\AA \mathrm{SiN}_{x}{ }^{1,3,5}$ An explanation for the high value in this work is the fact that a single-crystal TiN template layer does not have any surface cusps at the emerging grain or column boundaries (see Fig. 7 in Ref. 1). Such cusps would cause the subsequent $\mathrm{SiN}$ layer to grow unevenly and possibly also act as a preferential site for amorphous tissue formation. Hence, with a single-crystal template, the growth front should be more flat and thereby provide the possibility to stabilize the thickest c-SiN layers.

By changing bias and/or temperature, the mobility of the arriving species can be affected. With a higher mobility, lower-energy positions should be easier to reach, acting favorably on the lattice and interfacial structure. This is seen for the 40- $\AA$ TiN, 5- $\AA$ SiN multilayer deposited at $500{ }^{\circ} \mathrm{C}$, where the diffractogram exhibits clear Pendellösung fringes. For $T_{\mathrm{s}}=300{ }^{\circ} \mathrm{C}$, however, only SL peaks are observed (see Fig. 3). Hence, a higher temperature promotes higher-quality interfaces. At even higher temperature, $T_{\mathrm{s}}=700{ }^{\circ} \mathrm{C}$, we observe a higher degree of a-SiN ${ }_{x}$ which indicates that c-SiN is less stable at higher temperatures. Apparently, this mixture of a-SiN ${ }_{x}$ and $\mathrm{c}-\mathrm{SiN}$ is beneficial from a mechanical properties point of view because an increase in hardness compared to the films grown at lower temperatures is 
observed. Increasing the bias affects the hardness [Fig. 8(b)] through defect generation. When increasing the bias from floating potential to $V_{\mathrm{s}}=50-60 \mathrm{~V}$, the amount of point defects introduced in the lattice through collision cascades will increase. This causes an increase in compressive stresses and, subsequently, in hardness. Increasing the bias to $V_{\mathrm{s}}=50-60 \mathrm{~V}$ also causes a slight degradation of the SL peaks and fringes in the corresponding diffractogram, suggesting a degradation of the interfaces compared to the $-30-\mathrm{V}$ case. Hence, an intermediate bias should be used to achieve the highest crystal quality.

Decreasing the TiN layer thickness while maintaining the $\mathrm{SiN}_{x}$ layer thickness constant will further decrease the amount of space dislocation that can be used for propagation before an interface is approached. Thus the superlattice hardening is affected ${ }^{32}$ as plastic flow is effectively hindered through differences in elastic modulus, coherency strains, and Hall-Petch effects. This results in a $\sim 100 \%$ hardness increase for samples with 20- $\AA$ TiN compared to monolithic TiN films.

\section{CONCLUSIONS}

Multilayer films of transitional metal nitride TiN and covalently bonded $\mathrm{SiN}_{x}$ have been grown by codeposition of $\mathrm{Ti}$ and $\mathrm{Si}$ in an $\mathrm{Ar} / \mathrm{N}_{2}$ atmosphere. We present evidence for growth of high crystalline order singlecrystal TiN/SiN(001) superlattice films exhibiting high film uniformity, similar to what is usually observed in semiconductor related films. Through high-resolution TEM and Z-contrast STEM, a direct view of the stabilized layers is provided. The epitaxial nature is lost as growth of a-SiN ${ }_{x}(x \geqslant 1)$ is initiated. However, as shown through STEM, even for a $\mathrm{SiN}_{x}$ layer thickness of $13 \AA$, the initial atomic layers grow epitaxially. With formation of $\mathrm{a}-\mathrm{SiN}_{x}$, the $\mathrm{N}$ content in the coating increases as shown by ERDA. Also, the films grown are pure to the detection limit, with essentially no $\mathrm{O}$ present. The highest hardness values are observed within films exhibiting epitaxial $\mathrm{SiN}$ interlayers, but polycrystalline films also exhibit a higher hardness than do monolithic TiN and $\mathrm{SiN}_{x}$ films. Suggested hardening effects are Koehler hardening, coherency strains, and small crystallite sizes (when polycrystalline).

The Ti-Si-N system exhibits strong segregation between the constituent phases. Consequently, the binary compounds of this system have been targeted for the growth of super or potentially ultrahard nanocomposites. $^{6-8}$ The three-dimensional geometry of grains and tissue phases in these nanocomposites makes the microstructural observation, e.g., through TEM, challenging. In this study, through use of multilayers as a model system, not only can the interfacial thickness be precisely controlled, but the two-dimensional structure also allows for direct structural observation through TEM. The recent results presented herein and in Hultman et al. ${ }^{4}$ will have impacts on the interpretation of $\mathrm{TiN}-\mathrm{SiN}_{x}$ nanocomposites as the presence of c-SiN or any other strain-stabilized $\mathrm{SiN}_{x}$ polytype depending on the given TiN surface must be taken into consideration.

\section{ACKNOWLEDGMENTS}

Financial support by the Swedish Research Council [Vetenskapsrådet (V.R.)] and the Swedish Foundation for Strategic Research [Stiftelsen für Strategisk Forskning (S.S.F.)] is gratefully acknowledged. Dr. Wolfgang Bohne (Hahn-Meitner-Institut Berlin) is gratefully acknowledged for the ERDA measurements.

\section{REFERENCES}

1. H. Söderberg, J.M. Molina-Aldareguia, L. Hultman, and M. Odén: Nanostructure formation during deposition of $\mathrm{TiN} / \mathrm{SiN}_{x}$ nanomultilayer films deposited by reactive magnetron sputtering. $\underline{\text { J.Appl. }}$ Phvs. 97. 114327 (2005)

2. H. Söderberg, J.M. Molina-Aldareguia, T. Larsson, L. Hultman, and M. Odén: Epitaxial stabilization of cubic-SiN in TiN/SiN $_{x}$ multilayers. Appl. Phys. Lett. 88, 191902 (2006).

3. X. Hu, H. Xhang, J. Dai, G. Li, and M. Gu: Study of the superhardness mechanism of Ti-Si-N nanocomposite films: influence of the thickness of the $\mathrm{Si}_{3} \mathrm{~N}_{4}$ interfacial phase. J. Vac. Sci. Technol. A 23, 114 (2005).

4. L. Hultman, J. Bareño, A. Flink, H. Söderberg, K. Larsson, V. Petrova, M. Odén, J.E. Greene, and I. Petrov: Interface structure in superhard TiN-SiN nanolaminates and superlattices: Film growth and ab initio calculations. Phvs. Rev. B 75, 155437 (2007).

5. Y-H. Chen, K.W. Lee, W-A. Chiou, Y-W. Chung, and L.M. Keer: Synthesis and structure of smooth, superhard TiN/SiN ${ }_{x}$ multilayer coatings with an equiaxed microstructure. Surf. Coat. Technol. 146/147, 209 (2001).

6. S. Veprek and S. Reiprich: A concept for the design of novel superhard coatings. Thin Solid Films 268, 64 (1995).

7. S. Veprek, M.G.J. Veprek-Heijman, P. Karvankova, and J. Prochazka: Different approaches to superhard coatings and nanocomposites. Thin Solid Films 476, 1 (2005).

8. A. Niederhofer, T. Bolom, P. Nesládek, K. Moto, C. Eggs, D.S. Patil, and S. Veprek: The role of percolation threshold for the control of the hardness and thermal stability of super- and ultrahard nanocomposites. Surf. Coat. Technol. 146/147, 183 (2001).

9. JCPDS No. 4-829. International Center for Diffraction Data: ICDD, Swarthmore, PA, 2004.

10. JCPDS No. 38-1420. International Center for Diffraction Data: ICDD, Swarthmore, PA, 2004.

11. H. Ljungcrantz, M. Odén, L. Hultman, J.E. Greene, and J-E. Sundgren: Nanoindentation studies of single-crystal (001), (011), and (111) oriented TiN layers on MgO. J. Appl. Phvs. 80. 6725 (1996).

12. C. Engström, T. Berlind, J. Birch, L. Hultman, I.P. Ivanov, S.R. Kirkpatrick, and S.L. Rohde: Design, plasma studies, and ion assisted thin film growth in unbalanced dual target sputtering system with a solenoid coil. Vacuum 56, 107 (2000).

13. H. Söderberg, J. Birch, L. Hultman, and M. Odén: RHEED studies during growth of TiN/SiN $/$ TiN trilayers on $\mathrm{MgO}(001)$. Surf. Sci. 601, 2352 (2007). 
14. C.R. Powell, N-E. Lee, Y-W. Kim, and J.E. Greene: Heteroepitaxial wurtzite and zinc-blende structure $\mathrm{GaN}$ grown by reactiveion molecular-beam epitaxy: Growth kinetics, microstructure, and properties. J. Appl. Phvs. 73, 189 (1993).

15. D.K. Aswal, K.P. Muthe, S. Tawde, S. Chodhury, N. Bagkar, A. Singh, S.K. Gupta, and J.V. Yakhmi: XPS and AFM investigations of annealing induced surface modifications of $\mathrm{MgO}$ single crystals. J. Crvst. Growth 236, 661 (2002).

16. J.M. Molina-Aldareguia: Processing and nanoindentation behaviour of nitride multilayers. Ph.D. Thesis, University of Cambridge, Cambridge, England, 2002.

17. D.L. Windt: IMD—Software for modeling the optical properties of multilayer films. Comput. Phvs. 12, 360 (1998).

18. M.S. del Rio and R.J. Dejus: XOP: Recent developments. Proc. SPIE 3448, 340 (1998).

19. W. Bohne, J. Röhrich, and G. Röschert: The Berlin time-of-flight ERDA setup. Nucl. Instrum. Methods Phys. Res., Sect. B 136-138, 633 (1998).

20. W.C. Oliver and G.M. Pharr: An improved technique for determining hardness and elastic modulus using load and displacement sensing indentation experiments. J. Mater. Res. 7, 1564 (1992).

21. L. Tapfer and K. Ploog: X-ray interference in ultrathin epitaxial layers: A versatile method for the structural analysis of single quantum wells and heterointerfaces. Phvs. Rev. B 40,9802 (1989).

22. U. Helmersson, S. Todorova, L. Markert, S.A. Barnett, J-E. Sundgren, and J.E. Greene: Growth of single-crystal TiN/VN strained-layer superlattices with extremely high mechanical hardness. J. Appl. Phys. 62, 481 (1987).

23. J. $\mathrm{Xu}, \mathrm{G}$. $\mathrm{Li}$, and $\mathrm{M}$. Gu: The microstructure and mechanical properties of TaN/TiN and TaWN/TiN superlattice films. Thin Solid Films 370, 45 (2000).

24. M. Shinn, L. Hultman, and S.A. Barnett: Growth, structure, and microhardness of epitaxial TiN/NbN superlattices. J. Mater. Res. 7. 901 (1992).
25. H. Söderberg, F. Giuliani, L. Hultman, W.J. Clegg, and M. Odén: Deformation structures in superhard TiN/SiNx nanolaminates. Thin Solid Films (2006, submitted).

26. S. Sambasivan and W.T. Petuskey: Phase chemistry in the Ti-Si-N system: Thermochemical review with phase stability diagrams. J. Mater. Res. 9, 2362 (1994).

27. A. Flink, T. Larsson, J. Sjölén, L. Karlsson, and L. Hultman: Influence of $\mathrm{Si}$ on the microstructure of arc evaporated $(\mathrm{Ti}, \mathrm{Si}) \mathrm{N}$ thin films; Evidence for cubic solid solutions and their thermal stability. Surf. Coat. Technol. 200, 1535 (2005).

28. L. Tapfer and K. Ploog: Improved assessment of structural properties of $\mathrm{Al}_{x} \mathrm{Ga}_{1-x} \mathrm{As} / \mathrm{GaAs}$ heterostructures and superlattices by double-crystal x-ray diffraction. Phvs. Rev. B 33, 5565 (1986).

29. M.W. Dashiell, J. Kolodzey, P. Boucaud, V. Yam, and J-M. Lourtioz: Heterostructures of pseudomorphic $\mathrm{Ge}_{1-y} \mathrm{C}_{y}$ and $\mathrm{Ge}_{1-x-y} \mathrm{Si}_{x} \mathrm{C}_{y}$ alloys grown on $\mathrm{Ge}(001)$ substrates. J. Vac. Sci. Technol., A 18. 1728 (2000).

30. G. Kioseoglou, S. Kim, Y.L. Soo, X. Chen, H. Luo, Y.H. Kao, Y. Sasaki, X. Liu, and J.K. Furdyna: Investigation of nanoscale structure in digital layers of $\mathrm{Mn} / \mathrm{GaAs}$ and $\mathrm{MnGa} / \mathrm{GaAs}$. Appl. Phvs. Lett. 80, 1150 (2002).

31. I.W. Kim, Q. Li, L.D. Marks, and S.A. Barnett: Critical thickness for transformation of epitaxially stabilized cubic AlN in superlattices. Appl. Phvs. Lett. 78, 892 (2001).

32. J.S. Koehler: Attempt to design a strong solid. Phvs. Rev. B 2, 547 (1970).

33. J.M. Cairney, R. Tsukano, M.J. Hoffman, and M. Yang: Degradation of TiN coatings under cyclic loading. Acta Mater. 52, 3229 (2004).

34. J.M. Cairney, M.J. Hoffman, P.R. Munroe, P.J. Martin, and A. Bendavid: Deformation and fracture of $\mathrm{Ti}-\mathrm{Si}-\mathrm{N}$ nanocomposite films. Thin Solid Films 479, 193 (2005). 